\title{
Inovação na Indústria de Transformação Brasileira: análise exploratória da série histórica dos dados da PINTEC
}

\author{
Innovation in the Brazilian Manufacturing Industry: an exploratory \\ analysis of the historical series of PINTEC data
}

\author{
André Luís Faria Duarte \\ ${ }^{1}$ Universidade do Grande Rio (UNIGRANRIO) e Comissão Nacional de Energia Nuclear (CNEN), Rio de Janeiro, RJ, Brasil
}

\begin{abstract}
Resumo
Este trabalho tem por objetivo destacar alguns pontos considerados importantes na evolução da inovação na indústria de transformação no Brasil, a partir de dados da Pesquisa de Inovação Tecnológica (PINTEC). O estudo tem caráter descritivo e exploratório e adotou pesquisa bibliográfica e documental, coletando dados secundários. A primeira parte da pesquisa explora os dados históricos da PINTEC relacionados à indústria de transformação, tendo como foco a evolução da taxa de inovação de produto e de processo. A segunda parte explora alguns dados da pesquisa de 2014, comparando-os com as de 2008 e 2011, relacionados às divisões da indústria de transformação que obtiveram as maiores taxas de inovação. A principal conclusão é a de que a indústria de transformação brasileira vinha, até 2008, inovando mais. Outra consideração é que as quatro divisões que apresentaram melhor desempenho na pesquisa de 2014 podem ser divididas em duas grandes áreas, chamadas de química/farmoquímica e eletro/eletrônicos.
\end{abstract}

Palavras-chave: Inovação. PINTEC. Indústria de transformação.

\begin{abstract}
This research aims to highlight some important points in the evolution of innovation in the Brazilian manufacturing industry, based on data from the Research for Technological Innovation. The study is descriptive and exploratory and has adopted bibliographic and documentary research, collecting secondary data. The first part of the research explores PINTEC's historical data related to the manufacturing industry, focusing on the evolution of the product and process innovation rate. The second part explores some data from the 2014 survey, comparing them with those of 2008 and 2011, related to the manufacturing industry divisions that achieved the highest innovation rates. The main conclusion is that the Brazilian manufacturing industry was, until 2008, innovating more. Another consideration is that the four divisions that performed best in the 2014 survey can be divided into two major areas, called chemistry/ pharmacology and electro/electronics.
\end{abstract}

Keywords: Innovation. PINTEC. Manufacturing industry.

Área Tecnológica: Inovação tecnológica. Gestão da inovação.

\section{Introdução}

Nos últimos anos, a inovação tem sido entendida como uma característica fundamental para a diferenciação das organizações e até mesmo uma questão de sobrevivência. Mais do que nunca, a prosperidade econômica está ligada a grandes avanços no desenvolvimento tecnológico, 
sendo a inovação, em função disso, um elemento essencial de uma sociedade moderna (VAN GILS; RUTJES, 2017). Em um mundo em constante mudança, com organizações competindo com todos na rede global, há muitos estudos sobre como criar diferencial em meio a inovações cada vez mais constantes (DICKEL; MOURA, 2016). De acordo com Aryanto, Fontana e Afiff (2015), a fim de sobreviver e incrementar o crescimento, as empresas devem continuamente criar inovações. Em uma sociedade baseada no conhecimento, as soluções inovadoras são imperativas e normalmente oferecem vantagens competitivas ao inovador (SCHWELLA, 2005). A inovação é extremamente relevante para a sustentabilidade das organizações, já que "[...] permite a sua sustentação tanto em termos de novos produtos, processos mais eficientes e baratos ou mesmo empresas mais aderentes às necessidades do ambiente" (CRUZ; FREZATTI; BIDO, 2015, p. 774).

$\mathrm{O}$ conceito de inovação, no entanto, tem suas raízes no início do século XX, por meio do trabalho de Joseph Schumpeter, economista austríaco nascido em 1883. Este autor lançou as bases do que se passou a considerar inovação, ressaltando a sua importância para o desenvolvimento econômico. Segundo Schumpeter (1988, p. 72), "[...] toda espécie de extensão de crédito para fins de 'inovações' é por definição a concessão de crédito ao empresário, e constitui um elemento do desenvolvimento econômico".

O Manual de Oslo, uma publicação da Organização para Cooperação Econômica e Desenvolvimento (OCDE), que objetiva orientar e padronizar conceitos, metodologias e construção de estatísticas e indicadores para interpretação de dados relacionados à ciência, tecnologia $e$ inovação, conceitua inovação como:

[...] a implementação de um produto (bem ou serviço) novo ou significativamente melhorado, ou um processo, ou um novo método de marketing, ou um novo método organizacional nas práticas de negócios, na organização do local de trabalho ou nas relações externas. (OCDE, 2007, p. 55)

Pode-se observar que a definição utilizada no Manual do Oslo é bastante abrangente, incluindo no conceito de inovação, a inovação em produtos, em processos, em marketing e em métodos organizacionais.

Autores, como Druker (1987), Hamel e Prahalad (1997), Castells (1999) e Hamel (2001), entre vários outros, já identificaram a necessidade da inovação por parte das organizações. $\mathrm{Na}$ realidade de um mundo globalizado e com um mercado hipercompetitivo, é cada vez mais uma questão de sobrevivência para as empresas o investimento em produtos e processos inovadores. Brunhera, Baço e Crotti (2015) sustentam que a inovação bem-sucedida não é o resultado apenas de inovação tecnológica, mas que depende da gestão de inovação, que inclui a mudança da forma organizacional, práticas e processos de uma empresa. Para Côrtes, Romano e Bastos Jr. (2011), é necessário um aprendizado constante para buscar, gerar e absorver a inovação. Pode-se dizer que a inovação é um processo fundamental de renovação da empresa, para modificar a forma como ela oferece e fornece seus produtos e serviços (SANTOS et al., 2015), sendo, portanto, um fator crítico na estratégia das organizações (FREZATTI et al., 2014).

Lima e Carvalho (2009) ressaltam que a inovação deve ser entendida como um processo interativo, construído a partir da contribuição de diferentes atores econômicos e sociais que possuem variados tipos de informações e conhecimentos. Para Bittar et al. (2014), a inovação tecnológica é constituída de novos produtos e processos, que impactam e são impactados por novos saberes. Nesse sentido, Pitassi (2011) sustenta que a inovação tecnológica não é um fato heroico, resultado do esforço isolado de uma empresa, mas sim de uma rede de aprendizado. 
Nesse contexto, a pesquisa tem o objetivo de destacar pontos importantes na evolução da inovação na indústria de transformação no Brasil, a partir de dados da Pesquisa de Inovação Tecnológica (PINTEC). A pesquisa se apresenta dividida em duas partes. A primeira explora os dados históricos da PINTEC relacionados à indústria de transformação, tendo como foco a evolução da taxa de inovação de produto e de processo. A segunda parte explora alguns dados da pesquisa de 2014, comparando-os com as de 2008 e 2011, relacionados às divisões da indústria de transformação que obtiveram as maiores taxas de inovação. O estudo se justifica em função da importância de se identificar o quanto a indústria de transformação brasileira tem aumentado, ou não, o grau de inovação de produtos e processos nos últimos anos, além de apontar as divisões da indústria que apresentam os melhores índices de inovação.

\section{Referencial Teórico}

A competitividade que surge pela inovação se mostra fundamental para o desempenho econômico de longo prazo de um país, na economia global baseada no conhecimento da atualidade (CARAYANNIS; GRIGOROUDIS, 2016). Atualmente, a busca por inovação é imperativa, principalmente em função da globalização (NASCIMENTO et al., 2018). De acordo com Sousa e Oliveira (2013), os países que mais avançaram no processo de inovação, o fizeram graças a vultuosos investimentos contínuos em pesquisa e desenvolvimento. Nesse sentido, com o objetivo de estimular o desenvolvimento econômico, os governos vêm formulando políticas públicas baseadas em iniciativas relacionadas ao apoio à inovação e ao empreendedorismo (FERNANDES et al., 2017). Dessa forma, cabe ressaltar a situação da inovação no Brasil, contextualizando-a historicamente, bem como entender a principal ferramenta de avaliação da inovação no país, a Pesquisa de Inovação Tecnológica (PINTEC).

\subsection{Inovação no Brasil}

O Brasil, desde a segunda metade do século XX, procurou estimular a pesquisa científica e tecnológica, criando, a partir do final da década de 1990, diversos mecanismos de financiamento no Sistema Nacional de Inovação (GOMES et al., 2015), procurando estimular a inovação em vários setores da economia. De acordo com Reis e Gomes (2017, p. 723), o país "[...] vem tentando montar e consolidar o seu Sistema Nacional-Regional de Inovação, para o fomento de atividades inovativas". Para Ribeiro e Porto (2015), tem sido positivo o esforço do governo em buscar fortalecer a competitividade através da inovação. Nesse sentido, percebe-se o aumento da importância dada à inovação tecnológica no país (GOMES; PEREIRA, 2015), apesar de não possuir a inovação em grande escala como motor do investimento (BIELSCHOWSKY, 2013).

De acordo com Côrtes, Romano e Bastos Jr. (2011), o país tem lançado mão de políticas sistemáticas de apoio à inovação, tendo, as empresas, oportunidade de se engajarem em atividades de inovação. Para esses autores, é grande o desafio da inovação no contexto brasileiro. Pires, Teixeira e Hastenreiter Filho (2012) ressaltam que o ambiente institucional de incentivo ao desenvolvimento industrial e à inovação exige articulação entre governo, indústria e universidade. Para Andreassi (2005), apesar de ser fundamental que o governo estabeleça políticas e programas de apoio, as empresas precisam fazer a sua parte, desenvolvendo programas, políticas e ações com o objetivo de incrementar a inovação, já que o "[...] aparato institucional 
é fundamental para a implantação do processo de inovação em uma organização" (SILVA et al., 2018, p. 760). Nesse ponto, Chaves (2013) argumenta que no Brasil, o crescimento de empreendimentos inovadores esbarra na carência da cultura de inovação. No entanto, para Bielschowsky (2013), a inovação poderá tornar-se um motor do desenvolvimento, caso o esforço científico e tecnológico no país tenha a intensidade desejada.

\subsection{Pesquisa PINTEC}

A Pesquisa de Inovação Tecnológica (PINTEC) é realizada pelo Instituto Brasileiro de Geografia e Estatística (IBGE), com o apoio da Financiadora de Estudos e Projetos (FINEP) e do Ministério da Ciência, Tecnologia e Inovação (MCTI). Tem como objetivo fornecer informações para a construção de indicadores relacionados às atividades de inovação tecnológica das empresas brasileiras.

A referência conceitual e metodológica da PINTEC é baseada na terceira edição do Manual Oslo (OCDE, 2007) e no modelo da Community Innovation Survey (CIS), versão 2008, proposto pela Oficina Estatística da Comunidade Europeia (Eurostat) (Statistical Office of the European Communities). Essa característica é importante, pois permite a comparação dos dados coletados com pesquisas equivalentes realizadas em outros países.

A PINTEC foi realizada nos anos 2000, 2003, 2005, 2008, 2011 e 2014. A partir da pesquisa de 2008, a PINTEC passou a divulgar os resultados de acordo com a nova Classificação Nacional de Atividades Econômicas (CNAE) 2.0, tendo como universo de investigação as atividades das indústrias extrativas e de transformação, serviços selecionados (edição, telecomunicações e informática) e Pesquisa e Desenvolvimento (P\&D). Vale ressaltar que os dados qualitativos, como as inovações de produto ou processo, referem-se ao período de três anos, incluindo-se o ano da pesquisa. Já os dados quantitativos, como pessoal ocupado, referem-se ao ano da pesquisa.

A PINTEC investiga a inovação de produto de forma independente da inovação de processo. Nesse sentido, cabe identificar, à luz do Manual de Oslo (OCDE, 2007), as definições desses dois tipos de inovação.

Uma inovação de produto é a introdução de um bem ou serviço novo ou significativamente melhorado no que concerne a suas características ou usos previstos. Incluem-se melhoramentos significativos em especificações técnicas, componentes e materiais, softwares incorporados, facilidade de uso ou outras características funcionais. (OCDE, 2007, p. 57)

Uma inovação de processo é a implementação de um método de produção ou distribuição novo ou significativamente melhorado. Incluem-se mudanças significativas em técnicas, equipamentos e/ou softwares. (OCDE, 2007, p. 58)

Além das inovações de produto e de processo, a partir de 2008 a PINTEC passou a investigar, também, as inovações organizacionais e de marketing.

A importância da PINTEC para o país, de acordo com Pinheiro (2013), pode ser observada em função da sua ampla utilização pela comunidade acadêmica, associações, empresas e órgãos governamentais de várias esferas, além de pautar, por meio de seus resultados, diversas políticas, especialmente de CT\&I, servindo como parâmetro para diversas metas da Estratégia Nacional de CT\&I (MCTI, 2012). 


\section{Metodologia}

A presente pesquisa tem caráter descritivo e exploratório, pois busca descrever e analisar um determinado fenômeno de forma abrangente, sem se preocupar em responder uma questão específica. A pesquisa exploratória não parte de uma hipótese específica a ser confirmada, mas tem o propósito de proporcionar familiaridade com um problema (GIL, 2008).

A pesquisa descritiva é desenvolvida, principalmente, no âmbito das ciências humanas e sociais (CERVO; BERVIAN; DA SILVA, 2007) e de acordo com Appolinário (2006), é um tipo de pesquisa que procura descrever uma realidade sem nela interferir.

Os procedimentos adotados foram a pesquisa bibliográfica e pesquisa documental. A pesquisa bibliográfica foi realizada, prioritariamente, a partir de livros de autores consagrados $e$ artigos científicos publicados em periódicos qualificados. A pesquisa documental foi feita com o acesso aos documentos relativos à pesquisa PINTEC do IBGE. A coleta de dados secundários foi realizada a partir desses documentos.

\section{Resultados e Discussão}

A verificação dos dados da PINTEC desde a sua primeira edição, em 2000, mostra a evolução no percentual de empresas da indústria de transformação brasileira que implementaram inovações, tendo diminuído, apenas, na pesquisa de 2011, tendo uma leve melhora de pesquisa de 2014. A queda em 2011 deveu-se principalmente à diminuição do percentual de empresas que implementaram inovações de produto, já que o percentual de empresas que implementaram inovações de processo manteve-se no patamar de $32 \%$, enquanto o percentual de empresas que implementaram inovações de produto diminuiu consideravelmente, apresentando um índice abaixo do obtido em 2000 (Gráfico 1).

Gráfico 1 - Percentual de empresas que implementaram inovações, inovações de produto e inovações de processo, no segmento da indústria de transformação

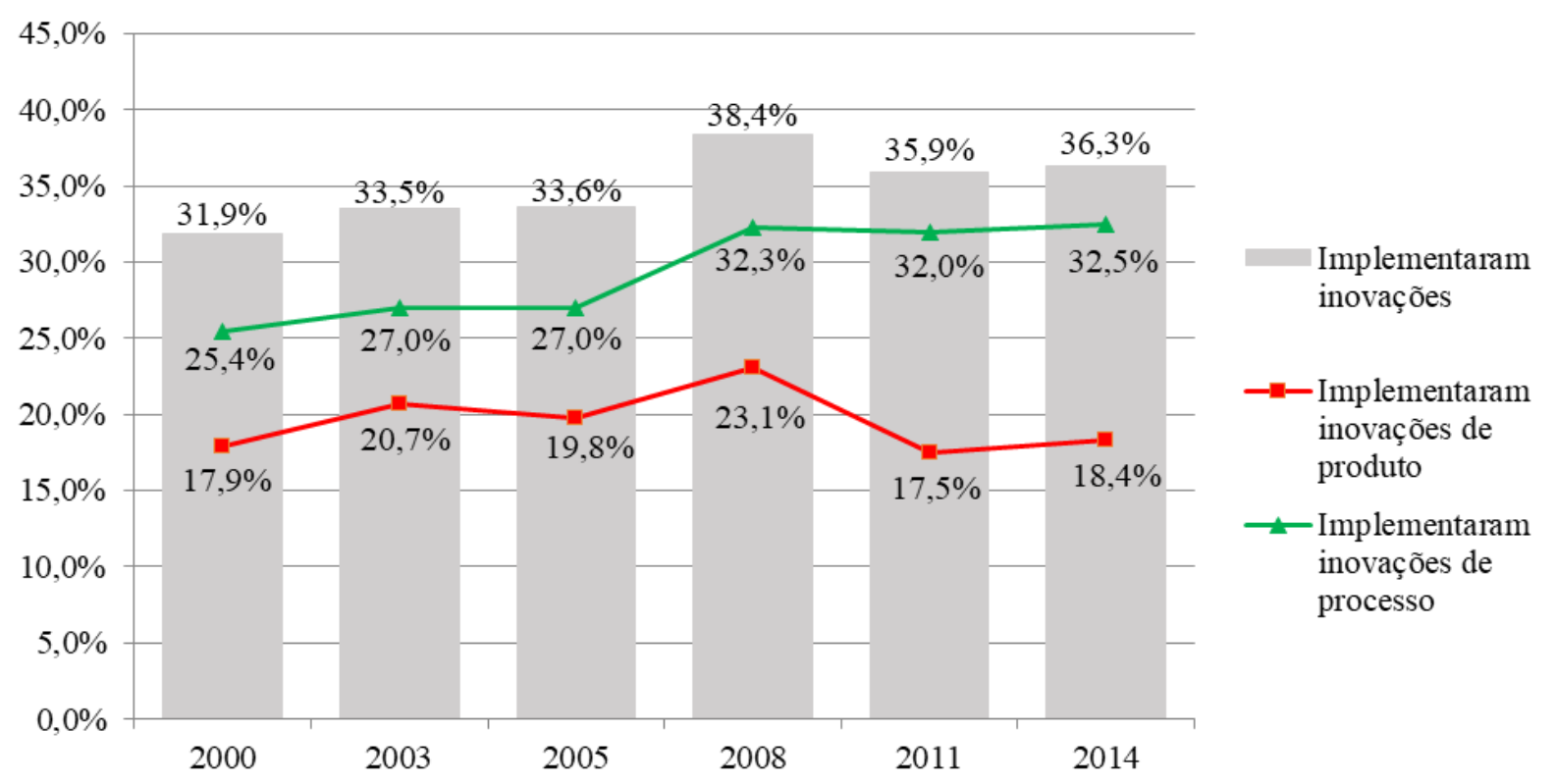

Fonte: Elaborado pelo autor deste artigo, com base nos dados da PINTEC - IBGE 
Pode-se observar que o percentual de empresas que implementaram inovação de processo tem sido maior que o de empresas que implementaram inovações de produto, desde a edição de 2000. Nas três primeiras pesquisas a diferença manteve-se por volta de 7 pontos percentuais. Na pesquisa de 2008, a diferença aumentou um pouco, para 9 pontos percentuais, e na de 2011 ficou em torno de 14 pontos percentuais, mantendo-se nesse patamar na pesquisa de 2014 .

Ao analisarmos esse índice considerando apenas o universo das empresas da indústria de transformação que implementaram inovações, o percentual de empresas que inovaram em processo se mantém estável nas três primeiras edições da PINTEC, e apresenta aumento consistente nas últimas duas edições, chegando ao índice de 89,0\% em 2011. Já o índice das empresas que inovaram em produto apresentou aumento na pesquisa de 2003, manteve-se mais ou menos estável nas três pesquisas seguintes, e apresentou forte queda na pesquisa de 2011, indo de 60,2\% em 2008 para 48,7\% em 2011 (Gráfico 2).

Gráfico 2 - Percentual de empresas que implementaram inovações de produto e inovações de processo, no segmento da indústria de transformação, dentre as que implementaram inovações

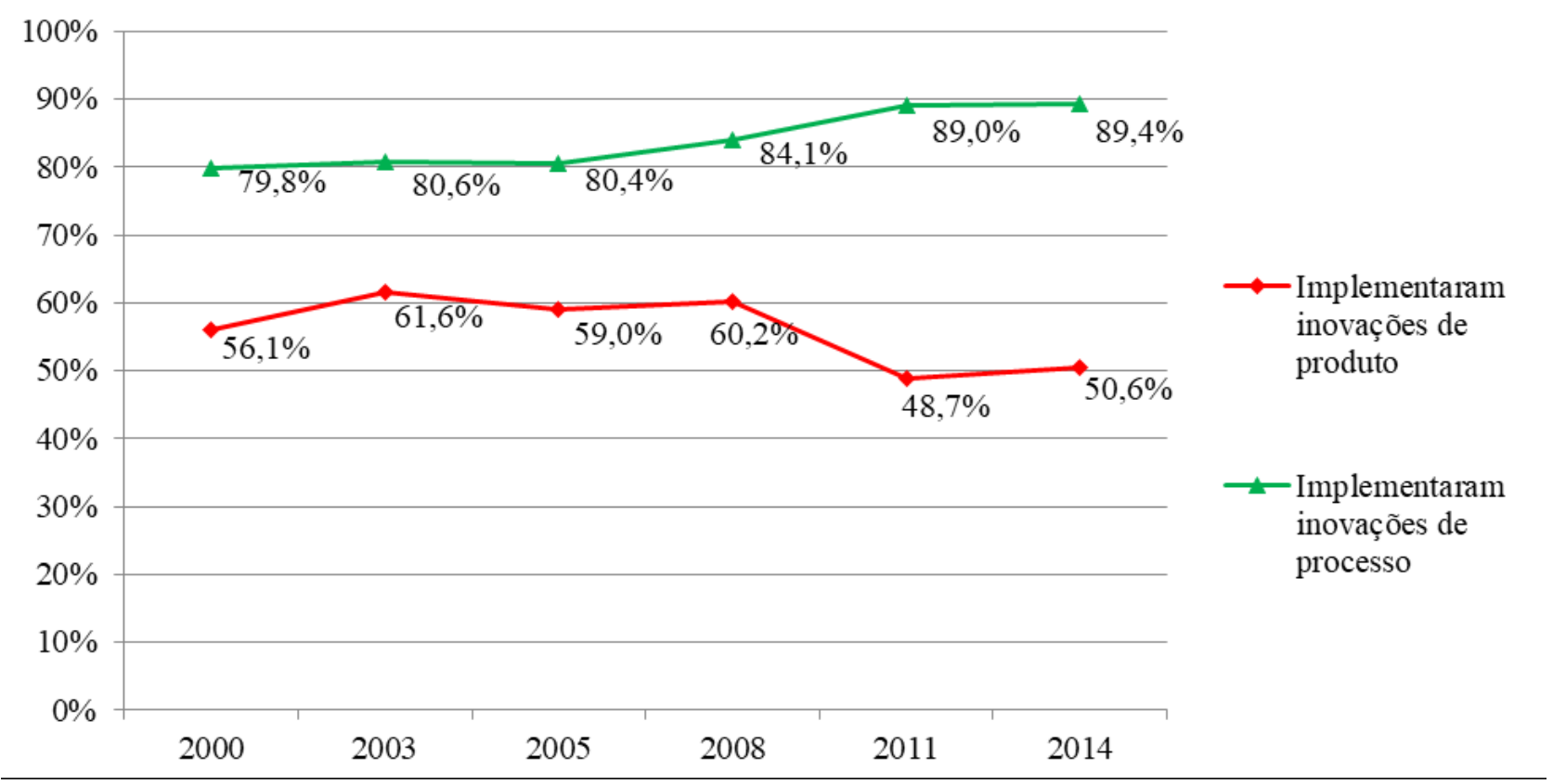

Fonte: Elaborado pelo autor deste capítulo, com base nos dados da PINTEC - IBGE

Na pesquisa de 2014, a divisão em que ocorreu o maior percentual de inovação foi a de fabricação de equipamentos de informática, produtos eletrônicos e ópticos. Essa divisão, desde a pesquisa de 2008, vem apresentando incremento no índice de inovação, apresentando 56,4\% em 2008, 59,2\% em 2011 e saltando para 68,2\% em 2014. Ao se analisar as inovações de produto, percebe-se que ocorreu um pequeno aumento no índice de 2008 para 2011, de 43,9\% para 45,8\%, e um grande salto para 59,0\% em 2014. Já as inovações de processo cresceram de forma regular nessa divisão, passando de 37,9\% em 2008 para 48,4\% em 2011 e para 59,0\% em 2014, mostrando um crescimento robusto e constante em torno de 10 pontos percentuais a cada triênio (Gráfico 3). Em 2014, essa divisão também foi a que apresentou o melhor índice tanto nas empresas que inovaram em produto quanto nas empresas que inovaram em processo. Vale ressaltar que essa divisão apresentou o terceiro maior índice na pesquisa de 2008, ficou em segundo lugar na pesquisa de 2011 e em primeiro lugar nesta de 2014. 
Gráfico 3 - Percentual de empresas que implementaram inovações, inovações de produto e inovações de processo, na divisão de Fabricação de equipamentos de informática, produtos eletrônicos e ópticos

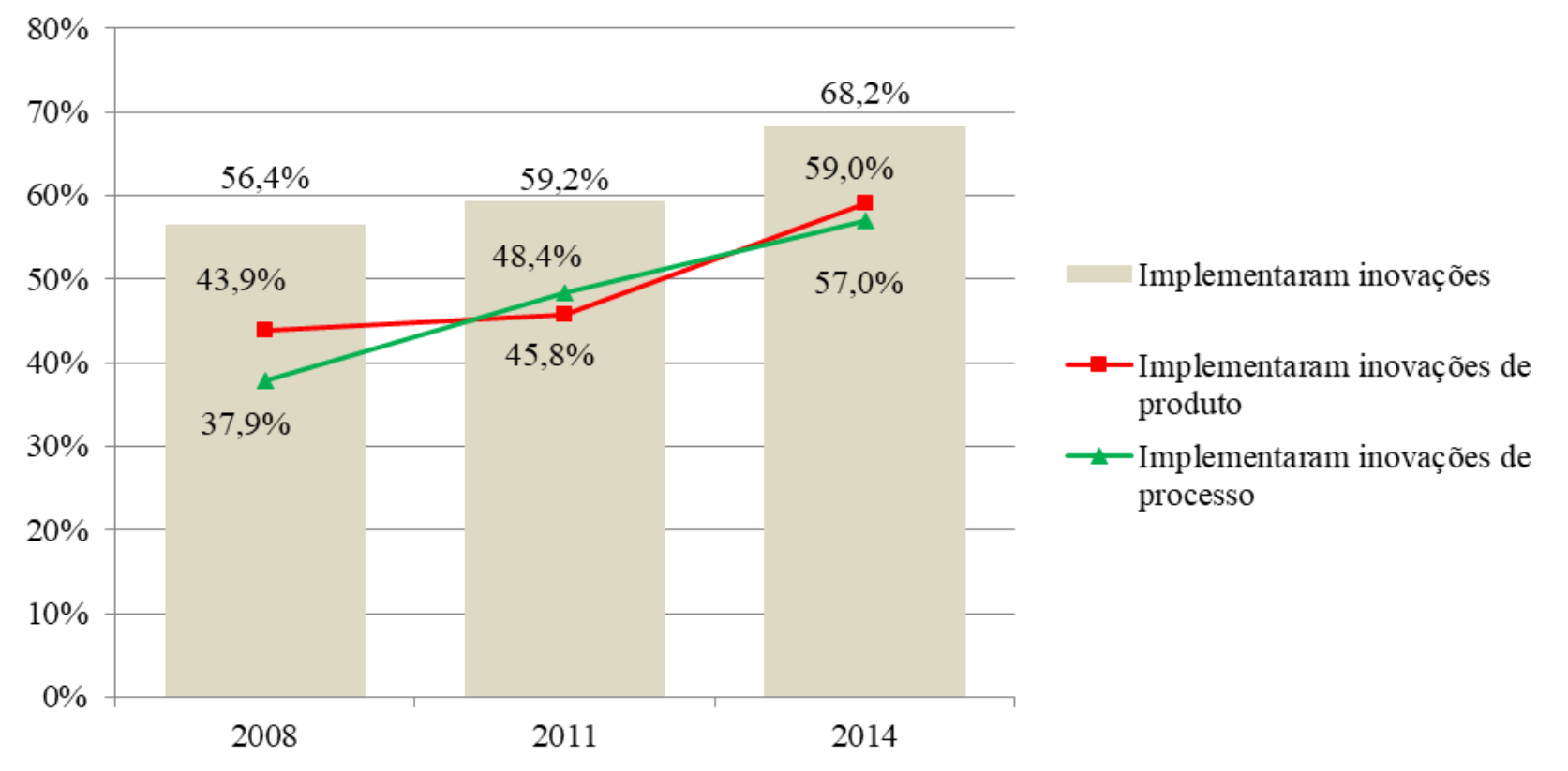

Fonte: Elaborado pelo autor deste artigo, com base nos dados da PINTEC - IBGE

A divisão em que ocorreu o segundo maior percentual de inovação na pesquisa de 2014 foi a de fabricação de produtos farmoquímicos e farmacêuticos, com $52,2 \%$ de ocorrência de inovações. Essa divisão apresentou comportamento inverso ao da divisão de fabricação de equipamentos de informática, produtos eletrônicos e ópticos, apresentando queda no índice nas três últimas edições da PINTEC, com 63,7\% na edição de 2008, 53,8\% na edição de 2011 e 52,2\% em 2014. Chama a atenção o fato de que em 2008 o número de empresas que implementaram inovações de produto foi maior do que o das que implementaram inovações de processo, ficando praticamente empatado em 2011 no patamar de $37 \%$ e se invertendo em 2014, com o índice das empresas que implementaram inovações de processo de 44,8\% e das que implementaram inovação de produto em 39,0\%. Em 2014, essa divisão foi também a que apresentou o segundo melhor índice tanto nas empresas que inovaram em produto quanto nas empresas que inovaram em processo. Outro ponto que chama a atenção nessa divisão é a diminuição do índice geral na pesquisa de 2014, enquanto os índices das empresas que implementaram inovações de produto e das empresas que implementaram inovações de processo aumentou. Isso se justifica pelo aumento do número de empresas que implementaram ambas 
(Gráfico 4). Essa divisão foi a que apresentou maior índice na pesquisa de 2008 e em 2011 ficou em quarto lugar.

Gráfico 4 - Percentual de empresas que implementaram inovações, inovações de produto e inovações de processo, na divisão de fabricação de produtos farmoquímicos e farmacêuticos

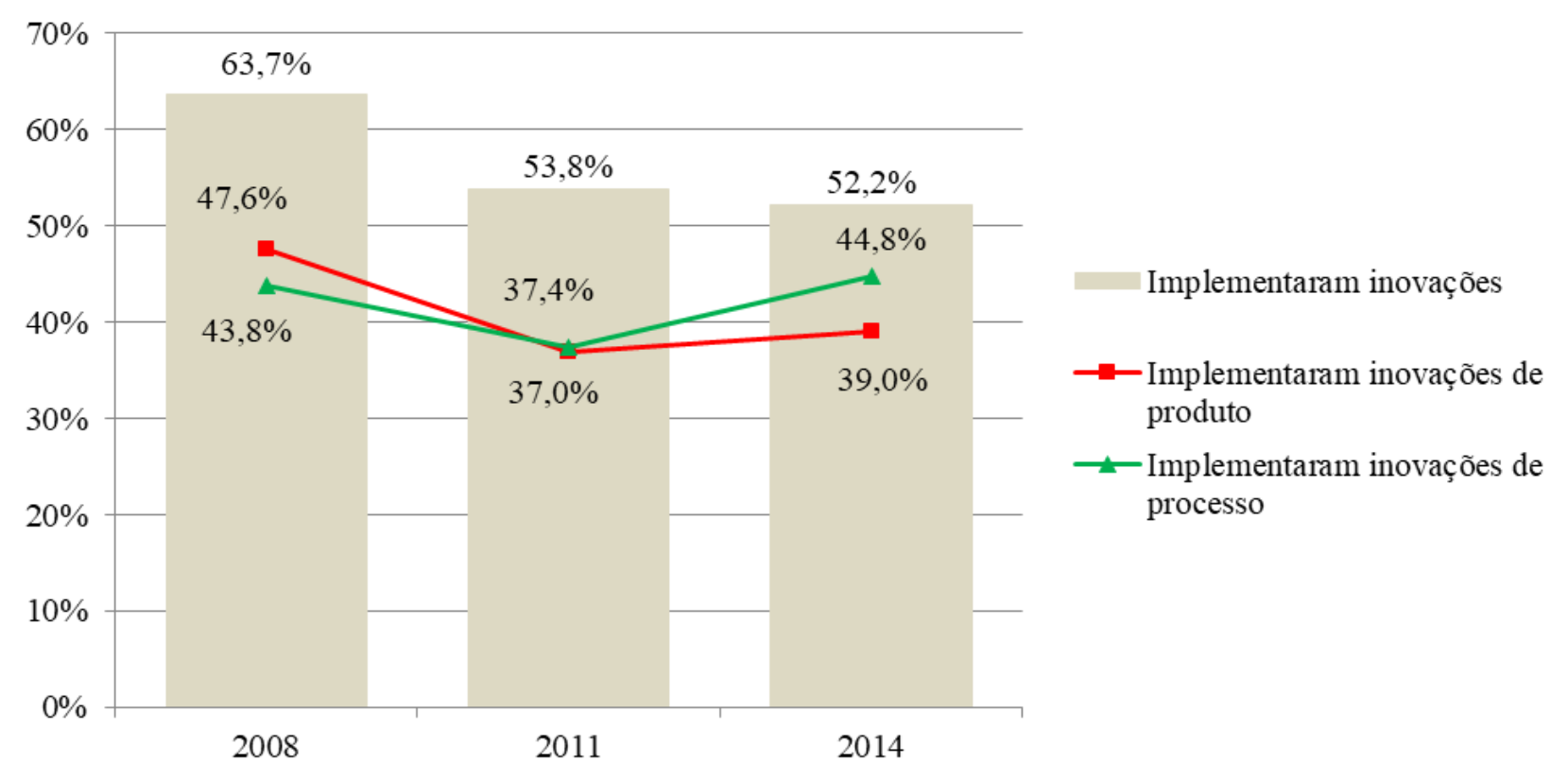

Fonte: Elaborado pelo autor deste artigo, com base nos dados da PINTEC - IBGE

A divisão de fabricação de produtos químicos aparece na terceira posição das divisões com maior percentual de empresas que implementaram inovações no triênio 2012-2014, com $49,6 \%$. Na pesquisa de 2008, referente ao triênio 2006-2008, essa divisão ficou em segundo lugar, com 58,1\%, aumentando para $59,1 \%$ na pesquisa de 2011 onde se posicionou em terceiro lugar. Ou seja, da pesquisa de 2011 para a de 2014 houve diminuição de quase 10 pontos percentuais no índice. Em relação às empresas que implementaram inovações em produto, o percentual vem diminuindo ao longo das últimas três edições da pesquisa, apresentando 45,2\% em 2008, 36,5\% em 2011 e 34,1\% em 2014. Nas inovações de processo, houve um grande aumento na pesquisa de 2011 em relação à de 2008 , passando de $42,7 \%$ para $53,7 \%$ e uma grande queda na de 2014 em relação à de 2011, diminuindo para 39,6\%. Ou seja, em 2008 o índice de empresas que inovaram em produto era maior do que o de empresas que inovaram em processo, porém bem próximos. Em 2011 a ordem se inverteu e com uma grande diferença para as empresas que inovaram em processos. Já em 2014 os índices voltaram a se aproximar, mas ainda com o índice das empresas que inovaram em processo superior ao das que inovaram em produto (Gráfico 5). 
Gráfico 5 - Percentual de empresas que implementaram inovações, inovações de produto e inovações de processo, na divisão de produtos químicos

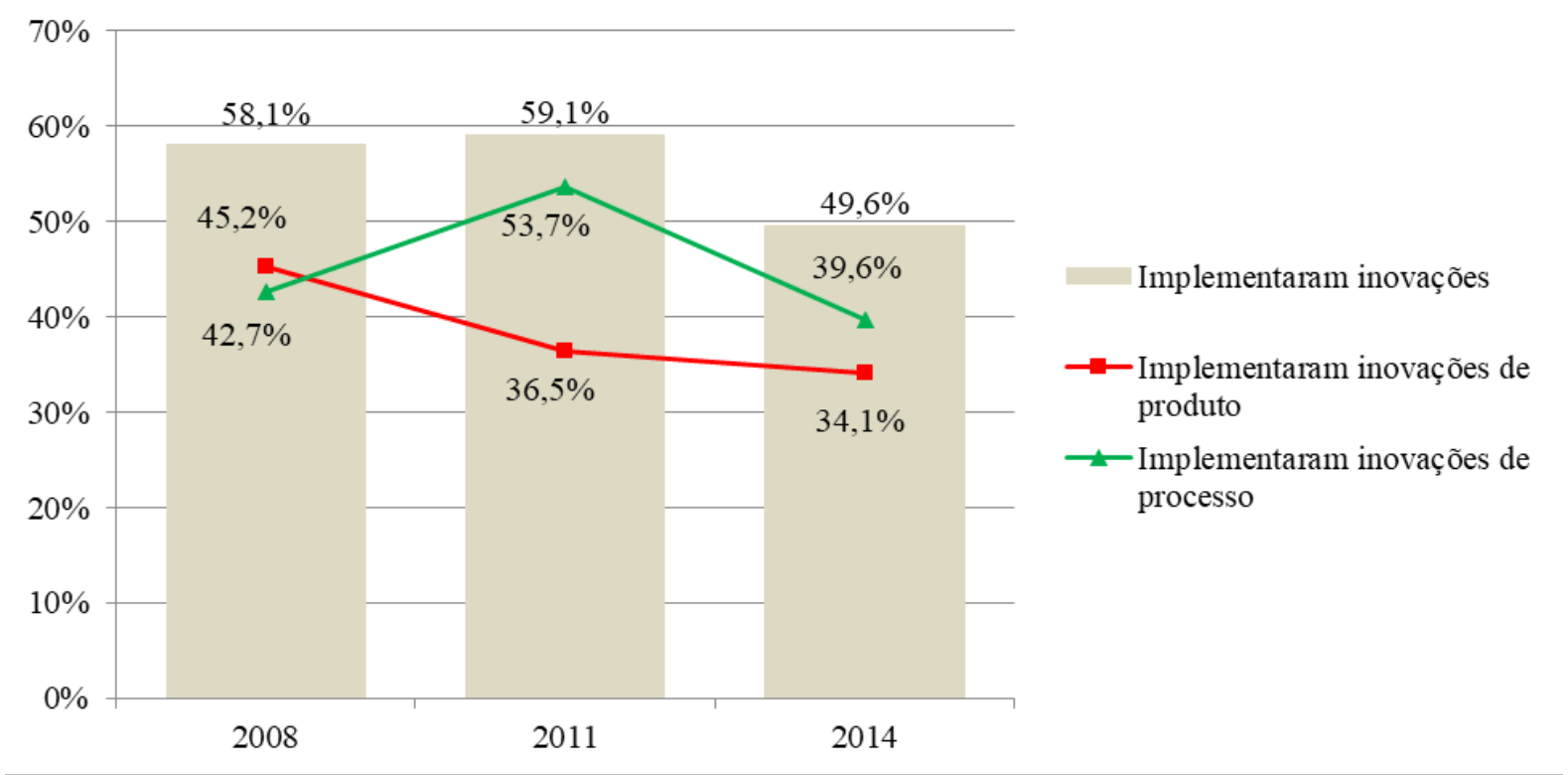

Fonte: Elaborado pelo autor deste artigo, com base nos dados da PINTEC - IBGE

Na quarta posição entre as divisões que tiveram maior percentual de empresas que implementaram inovações no triênio 2012-2014 ficou a divisão de fabricação de máquinas, aparelhos e materiais elétricos, com 47,1\%. No triênio 2006-2008 essa divisão apresentou o percentual de $46,5 \%$ com os índices das empresas que inovaram em produto e das que inovaram em processo bem próximos. No triênio 2009-2011, houve queda no índice para 44,3\%, com aumento no índice de empresas que inovaram em processo e diminuição no índice das que inovaram em produto. No triênio 2012-2014, tanto o índice das empresas que inovaram em produto quanto o das que inovaram em processo aumentaram (Gráfico 6).

Gráfico 6 - Percentual de empresas que implementaram inovações, inovações de produto e inovações de processo, na divisão de fabricação de máquinas, aparelhos e materiais elétricos

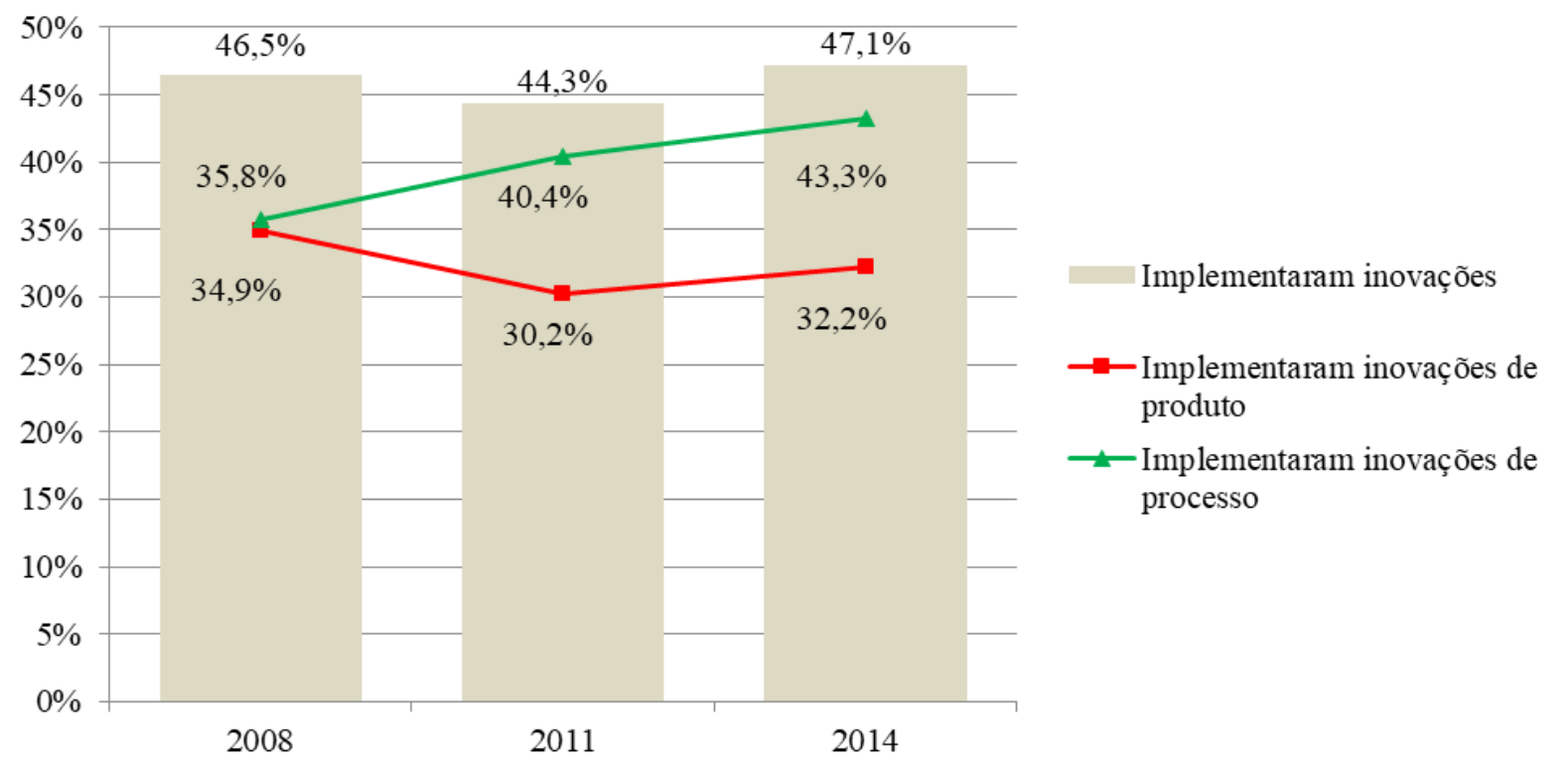

Fonte: Elaborado pelo autor deste artigo, com base nos dados da PINTEC - IBGE 
Uma observação importante que pode ser feita em relação à pesquisa de 2014 (Tabela 1), é que de todas as divisões, apenas duas divisões - a divisão de fabricação de equipamentos de informática, produtos eletrônicos e ópticos e a divisão de fabricação de outros equipamentos de transporte - apresentaram percentual de empresas que implementaram inovações de produto maior de que o percentual de empresas que implementaram inovações de processo. Ainda assim, nessas divisões os percentuais ficaram próximos.

Tabela 1 - Índice de inovação da indústria de transformação - 2014

\begin{tabular}{|c|c|c|c|}
\hline $\begin{array}{c}\text { DivisõES DA INDÚSTRIA DE TRANSFORMAÇÃo DE } \\
\text { ACORDO COM A CNAE } 2.0-2014\end{array}$ & $\begin{array}{c}\text { EMPRESAS QUE } \\
\text { IMPLEMENTARAM } \\
\text { INOVAÇÕES }\end{array}$ & $\begin{array}{c}\text { EMPRESAS QUE } \\
\text { IMPLEMENTARAM } \\
\text { INOVAÇÕES DE } \\
\text { PRODUTO }\end{array}$ & $\begin{array}{c}\text { EMPRESAS QUE } \\
\text { IMPLEMENTARAM } \\
\text { INOVAÇÕES DE } \\
\text { PROCESSO }\end{array}$ \\
\hline Indústrias de transformação (Total) & $36,30 \%$ & $18,45 \%$ & $32,50 \%$ \\
\hline $\begin{array}{l}\text { Fabricação de equipamentos de informática, } \\
\text { produtos eletrônicos e ópticos }\end{array}$ & $68,20 \%$ & $59,00 \%$ & $57,00 \%$ \\
\hline $\begin{array}{l}\text { Fabricação de produtos } \\
\text { farmoquímicos e farmacêuticos }\end{array}$ & $52,20 \%$ & $39,00 \%$ & $44,80 \%$ \\
\hline Fabricação de produtos químicos & $49,60 \%$ & $34,10 \%$ & $39,60 \%$ \\
\hline $\begin{array}{l}\text { Fabricação de máquinas, aparelhos } \\
\text { e materiais elétricos }\end{array}$ & $47,10 \%$ & $32,20 \%$ & $43,30 \%$ \\
\hline Fabricação de móveis & $44,00 \%$ & $21,60 \%$ & $41,30 \%$ \\
\hline Fabricação de bebidas & $43,70 \%$ & $17,20 \%$ & $40,40 \%$ \\
\hline Fabricação de produtos diversos & $42,60 \%$ & $23,80 \%$ & $39,20 \%$ \\
\hline $\begin{array}{l}\text { Fabricação de coque, de produtos derivados } \\
\text { do petróleo e de biocombustíveis }\end{array}$ & $42,30 \%$ & $18,10 \%$ & $39,60 \%$ \\
\hline Fabricação de artigos de borracha e plástico & $40,90 \%$ & $23,00 \%$ & $35,60 \%$ \\
\hline Fabricação de máquinas e equipamentos & $40,30 \%$ & $25,00 \%$ & $36,90 \%$ \\
\hline $\begin{array}{l}\text { Fabricação de veículos automotores, } \\
\text { reboques e carrocerias }\end{array}$ & $39,10 \%$ & $26,50 \%$ & $32,20 \%$ \\
\hline Fabricação de produtos de minerais não-metálicos & $38,50 \%$ & $15,70 \%$ & $35,70 \%$ \\
\hline Fabricação de outros equipamentos de transporte & $38,00 \%$ & $26,10 \%$ & $25,90 \%$ \\
\hline Metalurgia & $37,60 \%$ & $10,60 \%$ & $36,80 \%$ \\
\hline Fabricação de produtos do fumo & $35,00 \%$ & $18,80 \%$ & $30,30 \%$ \\
\hline Fabricação de produtos têxteis & $32,00 \%$ & $12,10 \%$ & $29,90 \%$ \\
\hline Fabricação de celulose, papel e produtos de papel & $30,30 \%$ & $10,90 \%$ & $29,40 \%$ \\
\hline Fabricação de produtos de metal & $29,40 \%$ & $13,50 \%$ & $26,10 \%$ \\
\hline Confecção de artigos do vestuário e acessórios & $29,00 \%$ & $11,30 \%$ & $25,20 \%$ \\
\hline $\begin{array}{l}\text { Preparação de couros e fabricação de artefatos } \\
\text { de couro, artigos de viagem e calçados }\end{array}$ & $28,80 \%$ & $16,20 \%$ & $23,80 \%$ \\
\hline Impressão e reprodução de gravações & $27,60 \%$ & $5,10 \%$ & $27,60 \%$ \\
\hline Fabricação de produtos de madeira & $25,30 \%$ & $12,90 \%$ & $24,70 \%$ \\
\hline $\begin{array}{l}\text { Manutenção, reparação e instalação } \\
\text { de máquinas e equipamentos }\end{array}$ & $22,90 \%$ & $8,50 \%$ & $20,70 \%$ \\
\hline
\end{tabular}

Fonte: Elaborado pelo autor deste artigo, com base nos dados da PINTEC - IBGE

Em relação à pesquisa de 2011 (Tabela 2), apenas a divisão de fabricação de máquinas e equipamentos apresentou percentual de empresas que implementaram inovações de produto 
maior de que o percentual de empresas que implementaram inovações de processo, com percentuais muito próximos: $29,5 \%$ e $29,3 \%$ respectivamente.

Tabela 2 - Índice de inovação da indústria de transformação - 2011

\begin{tabular}{|c|c|c|c|}
\hline $\begin{array}{l}\text { DivisóES DA INDÚSTRIA DE TRANSFORMAÇÃo DE ACOR- } \\
\text { DO COM A CNAE } 2.0-2011\end{array}$ & $\begin{array}{l}\text { EMPRESAS QUE } \\
\text { IMPLEMENTARAM } \\
\text { INOVAÇÕES }\end{array}$ & $\begin{array}{c}\text { EMPRESAS QUE } \\
\text { IMPLEMENTARAM } \\
\text { INOVAÇÕES DE } \\
\text { PRODUTO }\end{array}$ & $\begin{array}{c}\text { EMPRESAS QUE } \\
\text { IMPLEMENTARAM } \\
\text { INOVAÇÕES DE } \\
\text { PROCESSO }\end{array}$ \\
\hline Indústrias de transformação (Total) & $35,9 \%$ & $17,5 \%$ & $32,0 \%$ \\
\hline Fabricação de outros equipamentos de transporte & $65,3 \%$ & $14,2 \%$ & $63,5 \%$ \\
\hline $\begin{array}{c}\text { Fabricação de equipamentos de informática, } \\
\text { produtos eletrônicos e ópticos }\end{array}$ & $59,2 \%$ & $45,8 \%$ & $48,4 \%$ \\
\hline Fabricação de produtos químicos & $59,1 \%$ & $36,5 \%$ & $53,7 \%$ \\
\hline Fabricação de produtos farmoquímicos e farmacêuticos & $53,8 \%$ & $37,0 \%$ & $37,4 \%$ \\
\hline Fabricação de móveis & $44,6 \%$ & $18,7 \%$ & $37,4 \%$ \\
\hline $\begin{array}{l}\text { Fabricação de máquinas, aparelhos } \\
\text { e materiais elétricos }\end{array}$ & $44,3 \%$ & $30,2 \%$ & $40,4 \%$ \\
\hline Fabricação de celulose, papel e produtos de papel & $41,9 \%$ & $23,4 \%$ & $37,7 \%$ \\
\hline Fabricação de máquinas e equipamentos & $41,3 \%$ & $29,5 \%$ & $29,3 \%$ \\
\hline Metalurgia & $41,2 \%$ & $21,9 \%$ & $40,5 \%$ \\
\hline Fabricação de produtos alimentícios & $40,9 \%$ & $19,2 \%$ & $34,3 \%$ \\
\hline Impressão e reprodução de gravações & $39,1 \%$ & $18,3 \%$ & $38,9 \%$ \\
\hline $\begin{array}{l}\text { Fabricação de coque, de produtos derivados } \\
\text { do petróleo e de biocombustíveis }\end{array}$ & $38,1 \%$ & $14,0 \%$ & $37,1 \%$ \\
\hline Fabricação de artigos de borracha e plástico & $36,3 \%$ & $18,3 \%$ & $32,0 \%$ \\
\hline $\begin{array}{l}\text { Manutenção, reparação e instalação } \\
\text { de máquinas e equipamentos }\end{array}$ & $34,1 \%$ & $8,7 \%$ & $33,2 \%$ \\
\hline Fabricação de produtos diversos & $33,3 \%$ & $22,2 \%$ & $26,4 \%$ \\
\hline Fabricação de produtos de metal & $33,0 \%$ & $13,5 \%$ & $32,0 \%$ \\
\hline Confecção de artigos do vestuário e acessórios & $32,1 \%$ & $10,0 \%$ & $29,6 \%$ \\
\hline $\begin{array}{l}\text { Preparação de couros e fabricação de artefatos } \\
\text { de couro, artigos de viagem e calçados }\end{array}$ & $29,5 \%$ & $14,0 \%$ & $27,5 \%$ \\
\hline Fabricação de produtos de minerais não-metálicos & $29,2 \%$ & $17,4 \%$ & $25,6 \%$ \\
\hline $\begin{array}{l}\text { Fabricação de veículos automotores, } \\
\text { reboques e carrocerias }\end{array}$ & $29,1 \%$ & $21,5 \%$ & $25,8 \%$ \\
\hline Fabricação de produtos do fumo & $28,8 \%$ & $19,4 \%$ & $28,8 \%$ \\
\hline Fabricação de bebidas & $27,7 \%$ & $16,5 \%$ & $25,0 \%$ \\
\hline Fabricação de produtos têxteis & $26,6 \%$ & $8,6 \%$ & $25,6 \%$ \\
\hline Fabricação de produtos de madeira & $23,9 \%$ & $10,7 \%$ & $23,3 \%$ \\
\hline
\end{tabular}

Fonte: Elaborado pelo autor deste artigo, com base nos dados da PINTEC - IBGE 
Na pesquisa de 2008 (Tabela 3), foram quatro as divisões em que isso ocorreu: Fabricação de produtos do fumo, Fabricação de produtos químicos, Fabricação de produtos farmoquímicos e farmacêuticos e Fabricação de equipamentos de informática, produtos eletrônicos e ópticos. Este dado está em linha com o observado de forma geral, no qual as empresas da indústria de transformação brasileira têm implementado mais inovações de processo do que de produto.

Tabela 3 - Índice de inovação da indústria de transformação - 2008

\begin{tabular}{|c|c|c|c|}
\hline $\begin{array}{c}\text { DivisõES DA INDÚSTRIA DE TRANSFORMAÇÃo DE ACOR- } \\
\text { DO COM A CNAE } 2.0-2008\end{array}$ & $\begin{array}{c}\text { EMPRESAS QUE } \\
\text { IMPLEMENTARAM } \\
\text { INOVAÇÕES }\end{array}$ & $\begin{array}{c}\text { EMPRESAS QUE } \\
\text { IMPLEMENTARAM } \\
\text { INOVAÇÕES DE } \\
\text { PRODUTO }\end{array}$ & $\begin{array}{c}\text { EMPRESAS QUE } \\
\text { IMPLEMENTARAM } \\
\text { INOVAÇÕES DE } \\
\text { PROCESSO }\end{array}$ \\
\hline Indústrias de transformação (Total) & $38,4 \%$ & $23,1 \%$ & $32,3 \%$ \\
\hline Fabricação de produtos farmoquímicos e farmacêuticos & $63,7 \%$ & $47,6 \%$ & $43,8 \%$ \\
\hline Fabricação de produtos químicos & $58,1 \%$ & $45,2 \%$ & $42,7 \%$ \\
\hline $\begin{array}{l}\text { Fabricação de equipamentos de informática, } \\
\text { produtos eletrônicos e ópticos }\end{array}$ & $56,4 \%$ & $43,9 \%$ & $37,9 \%$ \\
\hline Fabricação de máquinas e equipamentos & $51,0 \%$ & $32,1 \%$ & $38,3 \%$ \\
\hline Impressão e reprodução de gravações & $47,2 \%$ & $19,8 \%$ & $45,5 \%$ \\
\hline $\begin{array}{l}\text { Fabricação de máquinas, aparelhos } \\
\text { e materiais elétricos }\end{array}$ & $46,5 \%$ & $34,9 \%$ & $35,8 \%$ \\
\hline $\begin{array}{l}\text { Fabricação de coque, de produtos derivados } \\
\text { do petróleo e de biocombustíveis }\end{array}$ & $45,9 \%$ & $22,5 \%$ & $43,0 \%$ \\
\hline $\begin{array}{l}\text { Fabricação de veículos automotores, } \\
\text { reboques e carrocerias }\end{array}$ & $45,1 \%$ & $30,4 \%$ & $37,5 \%$ \\
\hline Fabricação de produtos de metal & $39,6 \%$ & $19,4 \%$ & $34,8 \%$ \\
\hline Metalurgia & $39,5 \%$ & $20,5 \%$ & $32,6 \%$ \\
\hline Fabricação de produtos alimentícios & $38,2 \%$ & $25,2 \%$ & $31,4 \%$ \\
\hline $\begin{array}{l}\text { Preparação de couros e fabricação de artefatos } \\
\text { de couro, artigos para viagem e calçados }\end{array}$ & $36,8 \%$ & $24,4 \%$ & $32,5 \%$ \\
\hline Confecção de artigos do vestuário e acessórios & $36,8 \%$ & $19,4 \%$ & $33,4 \%$ \\
\hline Fabricação de artigos de borracha e plástico & $36,3 \%$ & $25,9 \%$ & $29,1 \%$ \\
\hline Fabricação de outros equipamentos de transporte & $36,1 \%$ & $15,0 \%$ & $33,0 \%$ \\
\hline Fabricação de produtos têxteis & $35,8 \%$ & $22,4 \%$ & $30,0 \%$ \\
\hline Fabricação de produtos diversos & $35,3 \%$ & $24,1 \%$ & $30,6 \%$ \\
\hline Fabricação de celulose, papel e produtos de papel & $35,2 \%$ & $25,5 \%$ & $34,0 \%$ \\
\hline Fabricação de bebidas & $34,6 \%$ & $22,1 \%$ & $27,4 \%$ \\
\hline Fabricação de móveis & $34,6 \%$ & $22,2 \%$ & $28,5 \%$ \\
\hline Fabricação de produtos de minerais não metálicos & $33,4 \%$ & $14,3 \%$ & $28,6 \%$ \\
\hline Fabricação de produtos do fumo & $26,5 \%$ & $19,6 \%$ & $17,7 \%$ \\
\hline $\begin{array}{l}\text { Manutenção, reparação e instalação } \\
\text { de máquinas e equipamentos }\end{array}$ & $25,9 \%$ & $15,6 \%$ & $22,1 \%$ \\
\hline Fabricação de produtos de madeira & $23,6 \%$ & $13,1 \%$ & $19,7 \%$ \\
\hline
\end{tabular}

Fonte: Elaborado pelo autor deste artigo, com base nos dados da PINTEC - IBGE 


\section{Considerações Finais}

Como pesquisa de caráter exploratório, o presente estudo não se propõe a apresentar conclusões categóricas ou exatas acerca do assunto estudado. O objetivo do estudo é o de promover insights que possam gerar futuras pesquisas específicas que objetivem confirmar ou não eventuais hipóteses.

A PINTEC é uma pesquisa extensa, sofisticada e que faz um levantamento bastante completo da inovação na indústria de todo o Brasil. Conforme já citado, os objetivos do presente estudo foram explorar os dados históricos da PINTEC nas suas seis edições, relacionados à indústria de transformação, com ênfase na taxa de inovação de produto e de processo das empresas; $e$ explorar alguns dados da pesquisa de 2014, comparando-os com a de 2008 e 2011, relacionados às divisões da indústria de transformação que obtiveram as maiores taxas de inovação na pesquisa de 2014.

A primeira consideração a ser feita ao analisar esses dados é que a indústria de transformação brasileira vinha, até 2008, de uma forma geral, inovando mais. A cada edição da pesquisa o índice de empresas que inovaram tanto em produto quanto em processos aumentava. No entanto, na pesquisa de 2011, esse índice caiu, de 38,4\% para 35,9\%, tendo uma pequena melhora para $36,3 \%$ na pesquisa de 2014 . Ou seja, a indústria de transformação brasileira está inovando menos do que no triênio 2006-2008.

Uma segunda consideração é que a indústria de transformação nacional inova mais em processo do que em produto, e que essa diferença aumentou bastante a partir da pesquisa de 2011, na qual o índice de inovações de processo se manteve no mesmo patamar e o índice de inovações de produto apresentou desempenho pior do que a primeira pesquisa, em 2000. Esses índices melhoraram pouco na pesquisa de 2014. O índice de empresas que implementaram inovações de processo atingiu o melhor percentual histórico na pesquisa, mas mantendo-se próximo das últimas pesquisas, no patamar de $32 \%$ e o índice de empresas que implementaram inovações de processo obteve melhora, mas continua bem abaixo do índice alcançado na pesquisa de 2008.

A terceira consideração é que as quatro divisões que apresentaram melhor desempenho podem ser divididas em duas grandes áreas, chamadas de química/farmoquímica e eletro/ eletrônicos. Essas áreas merecem uma atenção especial para que se identifique os motivos de estarem inovado mais, quando comparadas com outras.

Todas as considerações aqui apresentadas devem ser melhor analisadas a partir de estudos específicos, tanto qualitativos quanto quantitativos. A PINTEC é uma pesquisa que coleta um grande volume de dados e que gera a possibilidade de análises aprofundadas a partir de ferramentas mais robustas.

\section{Referências}

ANDREASSI, T. Ações internas voltadas ao fomento da inovação: as empresas também devem fazer sua "lição de casa". Cadernos EBAPE.BR, [S.l.], v. 3, n. SPE, p. 1-10, 2005.

APPOLINARIO, F. Metodologia da Ciência: Filosofia e Prática da Pesquisa. São Paulo: Cengage Learning, 2006. 
ARYANTO, R.; FONTANA, A.; AFIFF, A. Z. Strategic Human Resource Management, Innovation Capability and Performance: An Empirical Study in Indonesia Software Industry. Procedia-Social and Behavioral Sciences, [S.I.], v. 211, p. 874-879, 2015.

BIELSCHOWSKY, R. Estratégias de desenvolvimento e as três frentes de expansão no Brasil: um desenho conceitual. Rio de Janeiro: IPEA, 2013.

BITTAR, Angelo Antonio Mesquita et al. A dificuldade de pavimentar o caminho para a inovação tecnológica no Brasil: o retrato dos rankings, alguns indicadores e obstáculos à sua sustentabilidade.

Cadernos de Prospecção, Salvador, v. 7, n. 4, p. 472, 2014.

BRUNHERA, D. C. U.; BAÇO, F. M. B.; CROTTI, P. C. A Eficiência dos Processos de Inovação na Indústria de Transformação: uma análise no estado do Paraná. RAI - Revista de Administração e Inovação, [S.I.], v. 12, n. 4, p. 187-204, 2015.

CARAYANNIS, E. G.; GRIGOROUDIS, E. Using multiobjective mathematical programming to link national competitiveness, productivity, and innovation. Annals of Operations Research, [S.l.], v. 247, n. 2, p. 635-655, 2016.

CASTELLS, M. A sociedade em rede. São Paulo: Paz e Terra, 1999.

CERVO, A. L.; BERVIAN, P. A; DA SILVA, R. Metodologia Científica. 6. ed. São Paulo: Pearson Prentice Hall, 2007.

CHAVES, M. do P. S. R. Ciência, tecnologia e inovação para o desenvolvimento da Amazônia: experiência da Universidade Federal do Amazonas. Parcerias Estratégicas, Brasília, DF, Ed. Esp., v. 18, n. 36, p. 55-74, jan.-jun. 2013.

CÔRTES, A. M.; ROMANO, C. A.; BASTOS JR., P. A. Instrumentos de Apoio à Inovação Tecnológica no Paraná: Disponibilidade e Uso nas Empresas do Arranjo Produtivo Local (APL) de Software de Curitiba. Sistemas \& Gestão, [S.I.], v. 6, n. 4, p. 447-462, 2011.

CRUZ, A. P. C. da; FREZATTI, F.; BIDO, D. de S. Estilo de Liderança, Controle Gerencial e Inovação: Papel das Alavancas de Controle. RAC - Revista de Administração Contemporânea, Rio de Janeiro, v. 19, n. 6, p. 772-794, dez. 2015.

DICKEL, D. G.; MOURA, G. L. de. Organizational performance evaluation in intangible criteria: a model based on knowledge management and innovation management. RAI - Revista de Administração e Inovação, [S.1.], v. 13, n. 3, p. 211-220, 2016.

DRUCKER, P. F. Inovação e espírito empreendedor: prática e princípios. São Paulo: Editora Pioneira, 1987.

FERNANDES, C. C. et al. Strategic assets in technology-based incubators in Brazil. European Journal of Innovation Management, [S.I.], v. 20, n. 1, p. 153-170, 2017.

FREZATTI, F. et al. O papel do Balanced Scorecard na gestão da inovação. RAE - Revista de Administração de Empresas, [S.1.], v. 54, n. 4, p. 381-392, 2014.

GIL, A. C. Métodos e Técnicas de Pesquisa Social. 6. ed. São Paulo: Atlas, 2008.

GOMES, M. A. S.; PEREIRA, F. E. C. Hélice Tríplice: Um ensaio teórico sobre a relação Universidade-Empresa-Governo em busca da inovação. International Journal of Knowledge Engineering and Management (IJKEM), [S.I.], v. 4, n. 8, p. 136-155, 2015.

GOMES, V. C. et al. Os fundos setoriais e a redefiniçao do modelo de promoção de ciência, 
tecnologia e inovação no Brasil: uma análise à luz do CT-Agro. Revista de Administração, São Paulo, v. 50, n. 3, p. 353-368, set. 2015.

HAMEL, G. A era da revolução. HSM Management, [S.1.], n. 24, janeiro-fevereiro, p. 116-126, 2001.

HAMEL, G.; PRAHALAD, C. K. Competindo pelo futuro: estratégias inovadoras para obter o controle do seu setor e criar mercados de amanhã. Rio de Janeiro: Campus, 1997.

LIMA, C. R. M.; CARVALHO, L. S. Informação, comunicação e inovação: gestão da informação para inovação em uma organização complexa. Informação \& Informação, Londrina, v. 14, n. 2, p. 1-20, jul.-dez. 2009.

MCTI. Estratégia Nacional de Ciência, Tecnologia e Inovação 2012-2015. Brasília: MCTI, 2012. Disponível em: http://livroaberto.ibict.br/218981.pdf. Acesso em: 17 out. 2018.

NASCIMENTO, Ticiano Gomes do et al. Patentes e apropriação de valor da inovação: o caso da própolis. Cadernos de Prospecção, Salvador, v. 11, n. 1, p. 87-102, 2018.

OCDE - Organização para Cooperação Econômica e Desenvolvimento. Manual de Oslo: diretrizes para coleta e interpretação de dados sobre inovação. 3. ed. [S.l.]: FINP, 2007. Disponível em: http:// download.finep.gov.br/imprensa/manual_de_oslo.pdf. Acesso em: 17 out. 2018.

PINHEIRO, A. M. Pesquisa de inovação: um poderoso instrumento para diagnóstico, desenho, implementação e monitoramento de políticas na área de Ciência, Tecnologia e Inovação.

ComCiência, Campinas, n. 150, jul. 2013.

PIRES, A. M. de B.; TEIXEIRA, F. L. C.; HASTENREITER FILHO, H. N. Colaboração nas atividades de pesquisa desenvolvimento e inovação: o que nos ensina o Modelo de Centros e Redes de Excelência Petrobras/COPPE UFRJ? Organizações e Sociedade, [S.l.], v. 19, n. 62, p. 507-526, 2012.

PITASSI, C. O papel de um centro de P\&D em empresas de ramos tradicionais: o caso da UN de logística da Vale. Cadernos EBAPE.BR, Rio de Janeiro, v. 9, n. 2, junho de 2011.

REIS, Diego Araujo; GOMES, Iracema Machado de Aragão. Capital humano, intensidade da inovação na indústria e crescimento econômico no Brasil. Cadernos de Prospecção, Salvador, v. 10, n. 4, p. 721-737, 2017.

RIBEIRO, W. L.; PORTO, G. S. Análises das Redes de Inovação resultantes dos financiamentos dos Fundos Setoriais de Biotecnologia e Energia. Revista Brasileira de Gestão de Negócios, [S.l.], v. 17, n. 58, p. 1.426-1.443, outubro-dezembro, 2015.

SANTOS, J. H. de A. et al. Searching for a Path: A Bibliometric study on Innovation and Technological Capabilities. International Journal of Innovation - IJI, [S.I.], v. 3, n. 2, p. 54-66, nov. 2015.

SCHUMPETER, J. Teoria do Desenvolvimento Econômico. São Paulo: Nova Cultural, 1988.

SCHWELLA, E. Inovação no governo e no setor público: desafios e implicações para a liderança.

Revista do Serviço Público, [S.l.], v. 56, n. 3, p. 259-276, 2005.

SILVA, Edcleyton B. F. et al. Pesquisa \& Inovação: a Propriedade Intelectual do Estado de Minas Gerais. Cadernos de Prospecção, Salvador, v. 11, n. 3, p. 757-769, 2018.

SOUSA, D.; OLIVEIRA, R. V. O apoio do Estado ao Empreendedorismo Inovador: a experiência do 
Prime no Brasil. Revista Políticas Públicas, São Luís, v. 17, n. 2, p. 328-338, 2013.

VAN GILS, M. J. G. M.; RUTJES, F. P. J. T. Accelerating chemical start-ups in ecosystems: the need for biotopes. European Journal of Innovation Management, [S.I.], v. 20, n. 1, p. 135-152, 2017.

\section{Sobre o autor}

\section{André Luís Faria Duarte}

E-mail: alduarte@cnen.gov.br

Analista em C\&T na Comissão Nacional de Energia Nuclear, Mestre em Administração pelo MADE/UNESA. Doutorando em Administração na UNIGRANRIO.

Endereço profissional: R. Gal. Severiano, n. 90, Botafogo, Rio de Janeiro, RJ. CEP: 22290-901. 